\title{
An Architecture for Lightweight Service Discovery Protocol in MANET ${ }^{*}$
}

\author{
Byong-In Lim, Kee-Hyun Choi, and Dong-Ryeol Shin \\ School of Information and Communication Engineering, \\ Sungkyunkwan University \\ 440-746, Suwon, Korea, +82-31-290-7125 \\ \{1bi77, gyunee, drshin\}@ece.skku.ac.kr
}

\begin{abstract}
As the number of Internet services grows, it is becoming more important for the network users to be able to locate and utilize those services that are of interest to them. As a result, a service discovery protocols are coming to play an increasingly important role in highly dynamic networks. In this paper, we describe a new architecture for service discovery called LSD (Lightweight Service Discovery). The LSD is a peer-to-peer cache-based service discovery protocol for ad hoc environments. Through the LSD protocol, small handheld mobile devices with wireless connectivity can detect each other in their neighboring devices, and perceive whether particular shared services are available or not. In addition, the LSD solves the overhead problems which arise when traditional discovery protocols are applied to mobile ad hoc networks.
\end{abstract}

\section{Introduction}

As the number of Internet services grows, service discovery protocols are coming to play an increasingly important role in highly dynamic networks. Consequently, a number of service discovery protocols have been proposed for wired networks. Prominent among these [1] are the SLP, Salutation, UPnP and UDDI. These conventional approaches to service discovery protocols can be classified into two main categories. These systems were mostly designed for an administrated network context and are either based on mediator based discovery approaches (e.g. UDDI, Jini) or on maintaining a network-wide multicast tree based on direct or peer-to-peer discovery approaches (e.g. UPnP, Salutation, SLP, when operated without directory agents). In ad hoc networks, the provision for service discovery is mandatory since they are designed for irregular and unexpected changes in network topology. In a service discovery environment, basic services that are readily available like discovery and delivery in wired networks have to be re-designed in order to take into account the wireless network's properties. Furthermore, whenever the consumer rearranges the components in such a system, the system must automatically adapt its configuration as necessary. In order to satisfy these diverse requirements, we designed a modified framework based on the existing service discovery algorithms $[2,3]$ and

\footnotetext{
* This research was partially supported by a grant from the CUCN, Korea and Korea Science \& Engineering Foundation (R01-2004-000-10755-0).
} 
implemented a lightweight service discovery protocol suitable for ad-hoc environments.

The remainder of the paper is organized as follows. Section 2 discusses the LSD Architecture. Service Propagation Algorithm is described in section 3. Finally, this paper is concluded in section 4 .

\section{Design of LSD Architecture}

Figure 1 shows the LSD (Lightweight Service Discovery) architecture and corresponding components in a single mobile device.

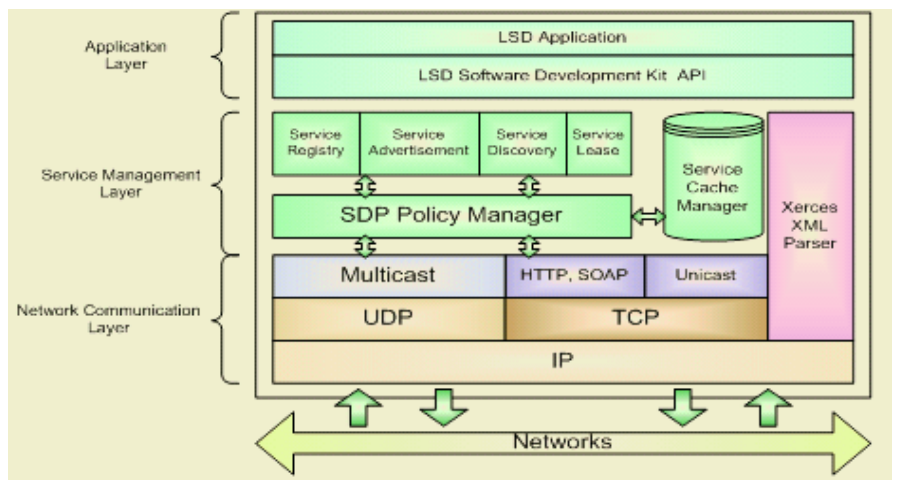

Fig. 1. LSD System Architecture

In Figure 1, the LSD consists of three layers: the application layer, the service management layer, and the network communication layer. The application layer provides the user with applications such as audio-video players and printing applications. The service management layer provides services associated with discovery. In the service management layer, we use a cache manager that registers information about the services offered by peers in the network. All of the devices in the network should listen to all advertisement messages, known as delta messages and save them in their local caches for a given period of time. When a user wants to find a particular service, they begin by searching for the service in their local caches at first. If no information is available locally, the user sends a request message through multicasting. The SDP Policy Manager in the layer is responsible for enforcing policies designed to control platform behavior. These policies are registered with the SDP Policy Manager which is responsible for ensuring that all components of the platform are in compliance with the specified policies. These policies can specify caching preferences such as the refresh rate or replacement strategy or describe advertisement preferences such as the frequency or time-to-live, etc. The network communication layer is associated with two protocols. The first is UDP which is used for multicasting; the second is TCP which is used unicasting. We send advertise/request messages by using UDP, and retrieve the data using http or soap based on TCP. 


\section{Service Propagation Algorithm of LSD}

The LSD pays attention to an efficient service discovery to exploit the nature of highly dynamic ad-hoc networks. Based on the Konark [3], it has developed a new algorithm which attempts to balance the convergence time and network bandwidth. The key difference between Gossip-Konark and LSD is service data structure and the convergence algorithm involved in joining a network. The Gossip-Konark has a structure of tree-based hierarchal services. On the other hand, the LSD has a structure of flat services that have a difference in patterns and message procedures of Query, Advertisement. Moreover, the LSD uses a dummy advertisement messages. When a node joins to new network, if it has no local service, it sends the dummy advertisement message. When a node receives a service message, it sends the difference between its relevant services and service information in the received service messages, called a delta message. Thus network overload is reduced. It also avoids the storm of concurrent multicasts by randomly assigning the multicast time interval. When a node receives the advertisement message, the LSD prevents multicast storm problem using RNDWAIT time similar to the Konark. Moreover, if the node which receives the service message verifies that its service registry has the same information as that in the received service message, it then remains silent and no multicasts occur. Figure 2 shows the message transmission procedure for the LSD. The incremental discovery procedure is able to reduce network traffic for service discovery due to delta messages.

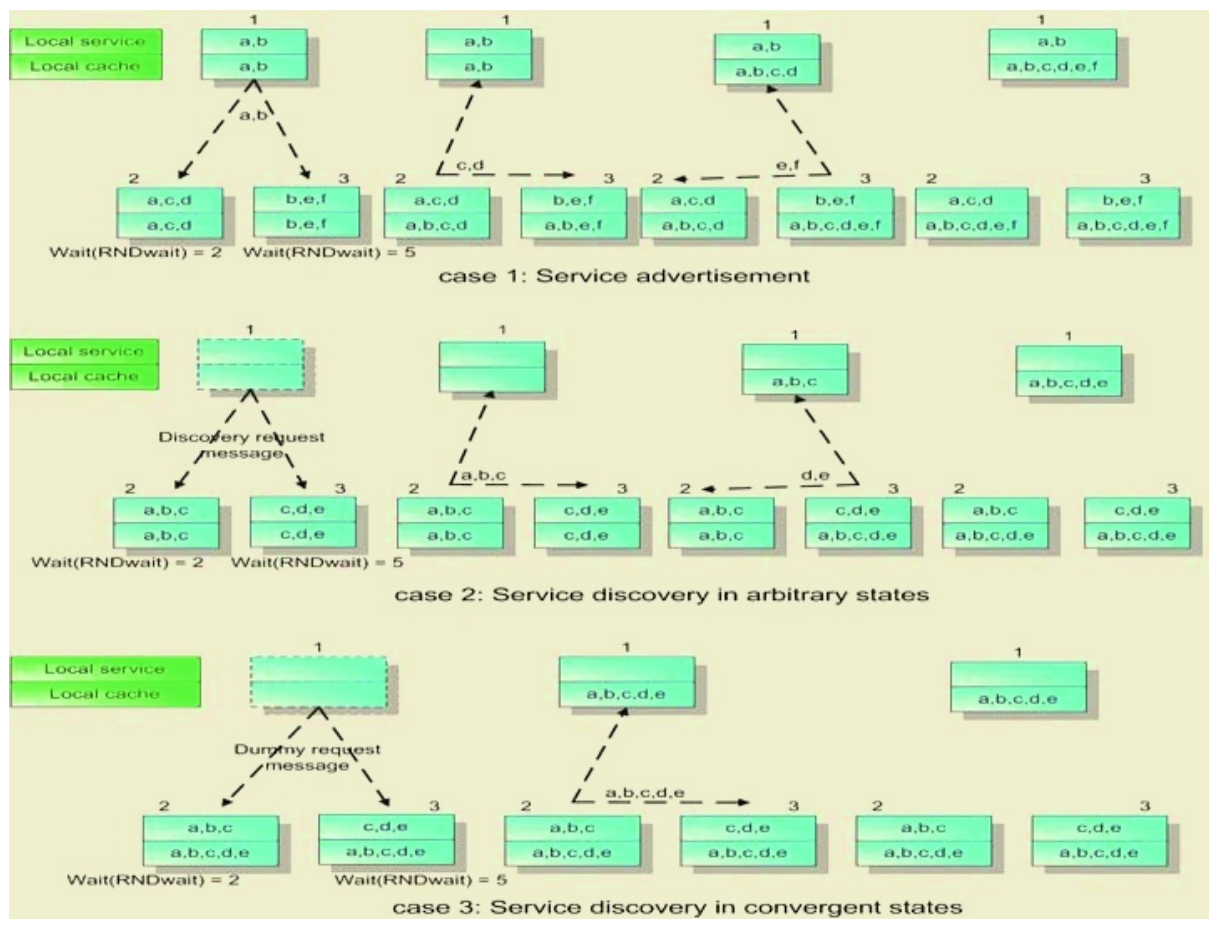

Fig. 2. Service Propagation Algorithm in LSD 
Suppose that an ad-hoc network of interest consists of three nodes: node 1, node 2, and node 3 . Assume that node 2 and node 3 have random waiting times (RNDWAIT) of 2 and 5 seconds, respectively. We have three cases according to the different situations as illustrated in Figure 2.

Case 1: service advertisement

(a) Node 1 sends its own service advertisement message to other nodes using the multicast. At this time, node 2 can send delta message based on the advertisement earlier than node 3, because node 2 has RNDWAIT $=2$, whereas node 3 has RNDWAIT $=5$.

(b) Listening to advertisement and delta messages from other nodes, node 3 sends service e, f to other nodes, because node 3 already receives services a, b, c, d.

Case 2: service discovery in arbitrary states, i.e., non-convergent states

(a) When node 1 joins to a new network, node 1 sends out a dummy request messages to other nodes. From these messages, node 2 multicasts a delta message ahead of other nodes, for the same reason as the case 1 .

(b) Node 3 makes a delta message based on the request and then sends it to other nodes, which results in fast discovery.

Case 3: service discovery in convergent states (where all nodes know the service information within the network)

(a) When node 1 joins to a new network in convergent state, node 1 sends out a dummy request message to other nodes. Then node 2 can send delta message earlier than other nodes as illustrated as in case 1 and case 2.

(b) Since the network already reaches the convergence of service information, the number of multicasts and resulting traffic road is reduced. One dummy request message is enough to reach the convergent state.

\section{Conclusion}

Unfortunately existing approaches to service trading are not well suited for these highly dynamic topologies since they rely on centralized servers and have high communication costs due to periodic query flooding. In this paper, we proposed a novel scheme to correct and optimize discovery protocol in case of ad-hoc environments. Currently we are performing a simulation and doing a comprehensive performance test for our prototyped system.

\section{References}

1. F. Zhu, M. Mutka, and L. Ni, "Classification of Service Discovery in Pervasive Computing Environments," Michigan State University, East Lansing, available at http://www.cse.msu.edu/ zhufeng/ServiceDiscoverySurvey.pdf MSU-CSE-02-24.

2. M. Nidd, "Service discovery in DEAPspace," IEEE Pers. Commun., vol.8, pp. 39-45, Aug. 2001.

3. C. Lee, A. Helal, N. Desai, V. Verma, B. Arslan, "Konark: A System and Protocols for Device Independent, Peer-to-Peer Discovery and Delivery of Mobile Services," IEEE System. Man. Cybernetic., vol. 33, no. 6, pp. 682-696, Nov. 2003 